\title{
Enhancement of the spin Peltier effect in multilayers
}

\author{
K. Uchida, ${ }^{1,2,3,4,{ }^{*}}$ R. Iguchi, ${ }^{2, \dagger}$ S. Daimon,,${ }^{2,5}$ R. Ramos, ${ }^{5}$ A. Anadón,${ }^{6,7}$ I. Lucas, ${ }^{6,7,8}$ P. A. Algarabel, ${ }^{7,9}$ L. Morellón, ${ }^{6,7,8}$ \\ M. H. Aguirre, ${ }^{6,7,8,10}$ M. R. Ibarra, ${ }^{6,7,8,10}$ and E. Saitoh ${ }^{2,3,5,11}$ \\ ${ }^{1}$ National Institute for Materials Science, Tsukuba 305-0047, Japan \\ ${ }^{2}$ Institute for Materials Research, Tohoku University, Sendai 980-8577, Japan \\ ${ }^{3}$ Center for Spintronics Research Network, Tohoku University, Sendai 980-8577, Japan \\ ${ }^{4}$ PRESTO, Japan Science and Technology Agency, Saitama 332-0012, Japan \\ ${ }^{5}$ WPI Advanced Institute for Materials Research, Tohoku University, Sendai 980-8577, Japan \\ ${ }^{6}$ Instituto de Nanociencia de Aragón, Universidad de Zaragoza, E-50018 Zaragoza, Spain \\ ${ }^{7}$ Departamento de Física de la Materia Condensada, Universidad de Zaragoza, E-50009 Zaragoza, Spain \\ ${ }^{8}$ Fundación Instituto de Nanociencia de Aragón, E-50018 Zaragoza, Spain \\ ${ }^{9}$ Instituto de Ciencia de Materiales de Aragón, Universidad de Zaragoza and Consejo Superior de Investigaciones Científicas, \\ E-50009 Zaragoza, Spain \\ ${ }^{10}$ Laboratorio de Microscopías Avanzadas, Universidad de Zaragoza, E-50018 Zaragoza, Spain \\ ${ }^{11}$ Advanced Science Research Center, Japan Atomic Energy Agency, Tokai 319-1195, Japan \\ (Received 26 March 2017; revised manuscript received 8 May 2017; published 30 May 2017)
}

\begin{abstract}
The spin Peltier effect (SPE), heat-current generation as a result of spin-current injection, has been investigated in alternately stacked $\mathrm{Pt} / \mathrm{Fe}_{3} \mathrm{O}_{4}$ multilayer films. The temperature modulation induced by the SPE in the $\left[\mathrm{Pt} / \mathrm{Fe}_{3} \mathrm{O}_{4}\right] \times n$ films was found to be significantly enhanced with increasing the number of $\mathrm{Pt} / \mathrm{Fe}_{3} \mathrm{O}_{4}$ bilayers $n$. This SPE enhancement is much greater than that expected for a simple stack of independent $\mathrm{Pt} / \mathrm{Fe}_{3} \mathrm{O}_{4}$ bilayers. The observed $n$ dependence of the SPE can be explained by introducing spin-current redistribution in the multilayer films in the thickness direction, in a manner similar to the enhancement of the spin Seebeck effect in multilayers.
\end{abstract}

DOI: 10.1103/PhysRevB.95.184437

\section{INTRODUCTION}

Spintronics [1-4] and thermoelectric conversion [5-7] possess great potential for the development of next-generation energy-saving technologies. These two research fields have been integrated since the discovery of the spin Seebeck effect (SSE) [8-21]. The SSE refers to the generation of a spin current as a result of a heat current; when a temperature gradient is applied to a paramagnet $(\mathrm{P}) /$ ferromagnet $(\mathrm{F})$ junction, a spin current is generated across the $\mathrm{P} / \mathrm{F}$ interface and then converted into a measurable electric voltage in $\mathrm{P}$ via the spin-orbit interaction. Since the SSE is driven by thermally activated dynamics of magnons in F [22-27], it appears not only in conductors $[8,10,17]$ but also in insulators [9,11-16,18-20], enabling the utilization of insulators for thermoelectric generation $[13,21]$. This is the unique feature of the SSE, offering a new direction in thermoelectric conversion technologies.

Recently, research on the spintronics-based thermoelectric conversion has been expanded into the reciprocal process of the SSE: the spin Peltier effect (SPE) [28-32]. The SPE refers to the generation of a heat current as a result of a spin current flowing across the $\mathrm{P} / \mathrm{F}$ interface, which was observed in a junction comprising a ferrimagnetic insulator yttrium iron garnet (YIG) and a paramagnetic metal, e.g., $\mathrm{Pt}$ and $\mathrm{W}$, by means of microfabricated thermopile sensors [28] and lock-in thermography (LIT) [31,32]. The utilization of insulators for thermoelectric heating and cooling is a unique feature of the

\footnotetext{
*UCHIDA.Kenichi@nims.go.jp

${ }^{\dagger}$ Present address: National Institute for Materials Science, Tsukuba 305-0047, Japan.
}

SPE. However, compared to an enormous number of SSE studies, experimental research on the SPE is limited to a few studies, which are insufficient to clarify the behavior and mechanism of the SPE. In particular, the establishment of principles for enhancing the SPE is important to realize novel heat-pump and temperature-control technologies driven by spintronics.

Here, we focus on the recently revealed fact that the SSE is strongly enhanced in alternately stacked $\mathrm{P} / \mathrm{F}$ multilayer films with increasing the number of P/F bilayers [33-35]. In light of the reciprocal relation between the SSE and SPE, the SPE is also expected to be enhanced in the multilayer films. In the present study, we demonstrate this behavior; we find that the temperature modulation induced by the SPE in the $\mathrm{P} / \mathrm{F}$ multilayer films significantly and monotonically increases with increasing the number of P/F bilayers. This SPE enhancement is much greater than that expected for a simple stack of independent $\mathrm{P} / \mathrm{F}$ bilayers. We discuss a possible origin of the bilayer-number dependence of the SPE on the basis of analytical and numerical calculations.

\section{EXPERIMENTAL PROCEDURE}

The sample systems used in this study are highly crystalline $\left[\mathrm{Pt} / \mathrm{Fe}_{3} \mathrm{O}_{4}\right] \times n$ multilayer films with bilayer numbers of $n=$ 1, 6, and 12 [Fig. 1(a)]. The multilayer films are the same as those used for the SSE measurements reported by Ramos et al. $[33,34]$, obtained by sequential growth of bilayer junctions consisting of paramagnetic $\mathrm{Pt}$ and ferrimagnetic $\mathrm{Fe}_{3} \mathrm{O}_{4}$ films on 0.5 -mm-thick single-crystalline $\mathrm{MgO}(001)$ substrates. The $\mathrm{Pt}$ and $\mathrm{Fe}_{3} \mathrm{O}_{4}$ layers were respectively deposited by dc magnetron sputtering and pulsed laser deposition methods in the same ultrahigh-vacuum chamber. The thickness of each 
(a)

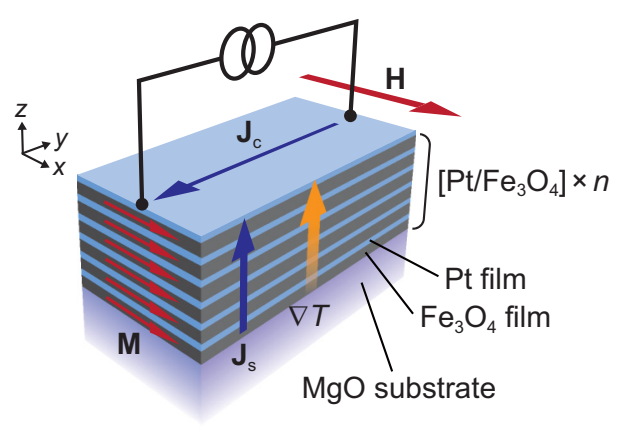

(b)

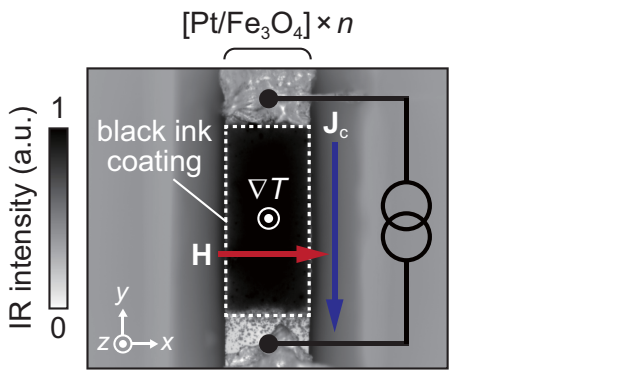

(c)

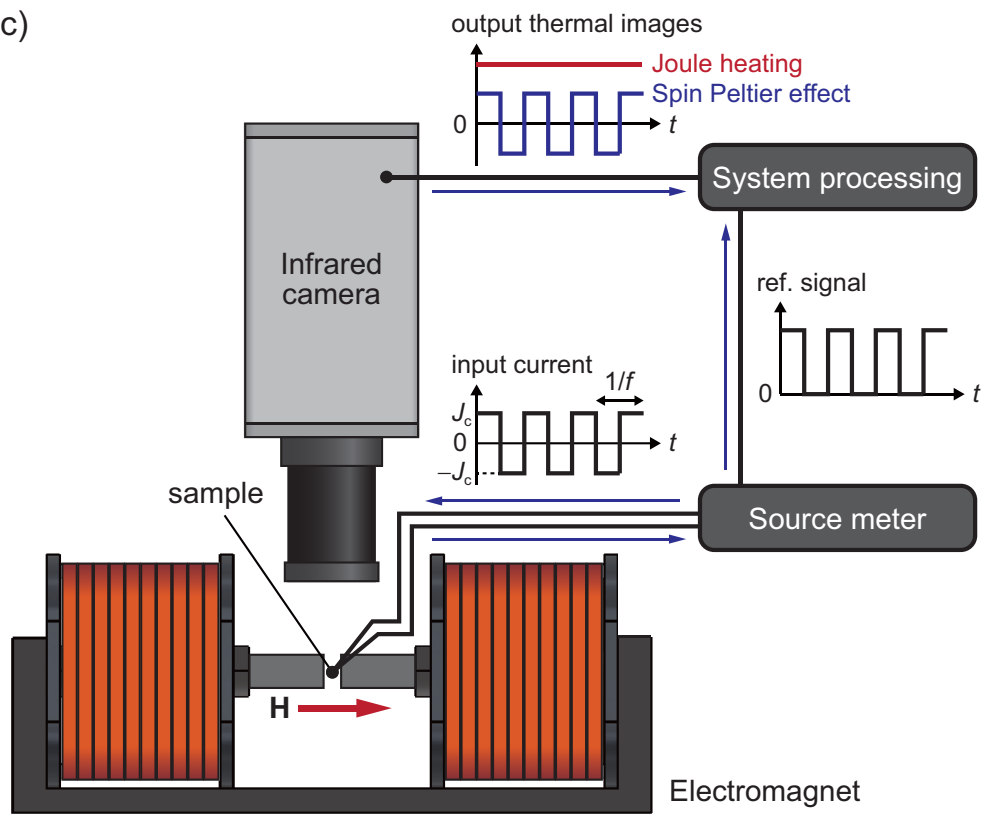

FIG. 1. (a) A schematic illustration of the $\left[\mathrm{Pt} / \mathrm{Fe}_{3} \mathrm{O}_{4}\right] \times n$ multilayer films. $\mathbf{H}, \mathbf{M}, \mathbf{J}_{\mathrm{c}}$, and $\mathbf{J}_{\mathrm{s}}$ denote the magnetic field vector with magnitude $H$, magnetization vector with magnitude $M$ of the $\mathrm{Fe}_{3} \mathrm{O}_{4}$ layers, charge current applied to the multilayer film, and spatial direction of the spin current generated by the spin Hall effect in the Pt layers, respectively. $\nabla T$ represents the temperature gradient appearing as a result of the heat current induced by the spin Peltier effect (SPE). (b) A steady-state infrared image of the $\left[\mathrm{Pt} / \mathrm{Fe}_{3} \mathrm{O}_{4}\right] \times n$ sample with the black-ink coating at thermal equilibrium. As an example, the image for $n=6$ is shown. (c) Lock-in thermography (LIT) for the SPE measurements. During the measurements, an ac charge current with rectangular wave modulation with amplitude $J_{\mathrm{c}}$ and frequency $f$ is applied to the multilayer film. $f$ is fixed at $5 \mathrm{~Hz}$ except for $f$-dependent measurements shown in Fig. 5.

$\mathrm{Pt}\left(\mathrm{Fe}_{3} \mathrm{O}_{4}\right)$ layer is $t_{\mathrm{P}}=7 \mathrm{~nm}\left(t_{\mathrm{F}}=23 \mathrm{~nm}\right)$. The length and width of the samples are $7 \mathrm{~mm}$ and $w=2 \mathrm{~mm}$, respectively. Although $\mathrm{Fe}_{3} \mathrm{O}_{4}$ is electrically conductive, it can be regarded as a ferrimagnetic insulator because the electrical conductivity of $\mathrm{Fe}_{3} \mathrm{O}_{4}$ is two orders of magnitude lower than that of Pt. Detailed information about the sample preparation and characterization is given in Refs. [33-35].

In the $\left[\mathrm{Pt} / \mathrm{Fe}_{3} \mathrm{O}_{4}\right] \times n$ multilayer films, the SPE is excited by the spin Hall effect (SHE) [36-39] in the Pt layers. When a charge current $\mathbf{J}_{\mathrm{c}}$ is applied to the Pt layers along the $y$ direction, the SHE generates a spin current along the $z$ direction. This spin current induces spin accumulation near the $\mathrm{Pt} / \mathrm{Fe}_{3} \mathrm{O}_{4}$ interfaces, of which the spin polarization is directed along the $x$ direction due to the symmetry of the SHE. This spin accumulation combines with magnetic moments in the $\mathrm{Fe}_{3} \mathrm{O}_{4}$ layers via the spin-mixing conductance [40]. Therefore, when the magnetization $\mathbf{M}$ of the $\mathrm{Fe}_{3} \mathrm{O}_{4}$ layers aligns along the $x$ direction, the spin-transfer torque across the $\mathrm{Pt} / \mathrm{Fe}_{3} \mathrm{O}_{4}$ interfaces induces a net energy transfer along the $z$ direction; this is the SPE $[28,31,32]$. The heat current generated by the SPE is proportional to the magnitude of the injected spin current and dependent on whether the spin-polarization direction in $\mathrm{Pt}$ is parallel or antiparallel to $\mathbf{M}$ of $\mathrm{Fe}_{3} \mathrm{O}_{4}$. The $\mathrm{SPE}$ in the $\left[\mathrm{Pt} / \mathrm{Fe}_{3} \mathrm{O}_{4}\right] \times n$ multilayer films can be measured by monitoring the spin-current-induced temperature change on the sample surface.

To observe the SPE in the $\left[\mathrm{Pt} / \mathrm{Fe}_{3} \mathrm{O}_{4}\right] \times n$ multilayer films, we employed the LIT method [41,42], which enables simple, versatile, and systematic measurements of the SPE [31,32]. The measurement procedure is summarized in Fig. 1(c). We measured the infrared radiation thermally emitted from the surface of the samples while applying an ac charge current with rectangular wave modulation with amplitude $J_{\mathrm{c}}$, frequency $f$, and no dc offset to the samples, where we attached electrical contacts near the ends of the top Pt layer and set $f=5 \mathrm{~Hz}$ except for $f$-dependent measurements shown in Appendix A. The obtained thermal images are transformed into the lock-in amplitude $A(>0)$ and phase $\phi\left(0^{\circ}<\phi<360^{\circ}\right)$ images by Fourier analysis; by extracting the first harmonic response of the thermal images, we can separate the SPE contribution $\left(\propto J_{\mathrm{c}}\right)$ from Joule-heating contribution $\left(\propto J_{\mathrm{c}}^{2}\right)$ because the Joule heating generated by the rectangular ac current is constant in time, as depicted in Fig. 1(c). The detected infrared intensity is converted into temperature information through the calibration method detailed in Supplementary Information of Ref. [31]. To enhance infrared emissivity and to ensure uniform emission properties, the sample surface was coated with insulating black ink, whose emissivity is $>0.95$; as shown in the steady-state infrared image of the sample under thermal equilibrium [Fig. 1(b)], the infrared intensity emitted from the black-ink-coated area near the center of the sample is much higher than that from the uncoated area near the electrical contacts. During the LIT measurements, an in-plane magnetic field $\mathbf{H}$ with magnitude $H$ was applied along the $x$ direction. When $|H|>1 \mathrm{kOe}, \mathbf{M}$ of the $\mathrm{Fe}_{3} \mathrm{O}_{4}$ layers is aligned along the $H$ direction. All the measurements were carried out at room temperature and atmospheric pressure. Here, we emphasize again that the electrical conductivity of $\mathrm{Fe}_{3} \mathrm{O}_{4}$ is two orders of magnitude lower than that of $\mathrm{Pt}$, and the charge current flows mainly in the Pt layers of the $\left[\mathrm{Pt} / \mathrm{Fe}_{3} \mathrm{O}_{4}\right] \times n$ multilayer 

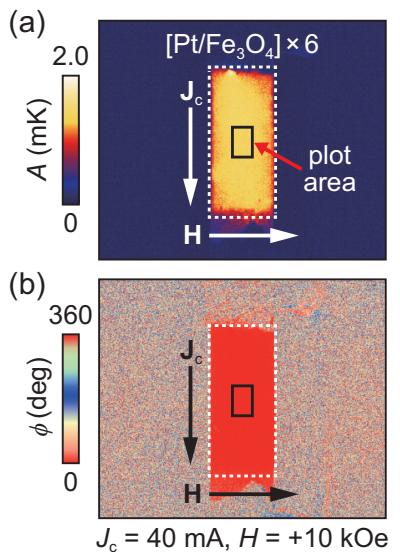
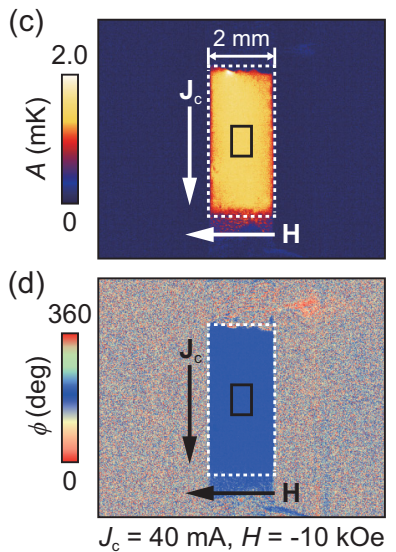
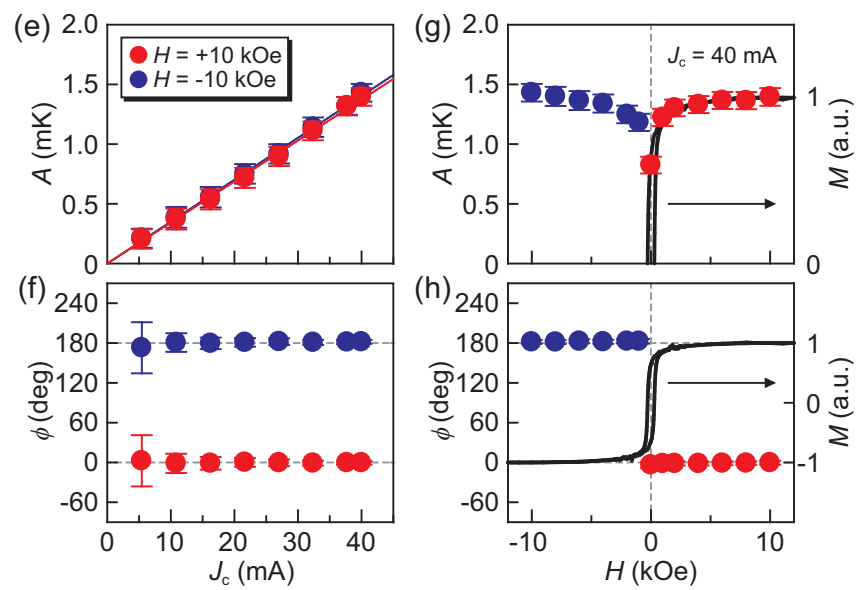

FIG. 2. (a) and (b) Lock-in amplitude $A$ and phase $\phi$ images for the $\left[\mathrm{Pt} / \mathrm{Fe}_{3} \mathrm{O}_{4}\right] \times 6$ sample at $J_{\mathrm{c}}=40 \mathrm{~mA}$ and $H=+10 \mathrm{kOe}$. (c) and (d) $A$ and $\phi$ images for the $\left[\mathrm{Pt} / \mathrm{Fe}_{3} \mathrm{O}_{4}\right] \times 6$ sample at $J_{\mathrm{c}}=40 \mathrm{~mA}$ and $H=-10 \mathrm{kOe}$. (e) and (f) $J_{\mathrm{c}}$ dependence of $A$ and $\phi$ for the $\left[\mathrm{Pt} / \mathrm{Fe} \mathrm{O}_{3}\right] \times 6$ sample at $H=+10$ or $-10 \mathrm{kOe}$. (g) and (h) $H$ dependence of $A$ and $\phi$ for the $\left[\mathrm{Pt} / \mathrm{Fe}_{3} \mathrm{O}_{4}\right] \times 6$ sample at $J_{\mathrm{c}}=40 \mathrm{~mA}$ and the $M-H$ curve of the sample.

films. Hereafter, for simplicity, the electrical conductivity and spin Hall angle of the Pt layers are assumed to be the same regardless of their position.

\section{RESULTS AND DISCUSSION}

\section{A. Observation of the spin Peltier effect in multilayers}

Figures 2(a) and 2(b) respectively show the $A$ and $\phi$ images for the $\left[\mathrm{Pt} / \mathrm{Fe}_{3} \mathrm{O}_{4}\right] \times 6$ sample at $J_{\mathrm{c}}=40 \mathrm{~mA}$ and $H=+10 \mathrm{kOe}(\mathbf{H} \|+x$ direction). The clear temperature modulation depending on the $J_{\mathrm{c}}$ direction appears on the sample surface with a lock-in phase of $\phi \sim 0^{\circ}$, meaning that the input charge current and output temperature modulation oscillate with the same phase under this condition. We found that the magnitude of the temperature modulation is proportional to $J_{\text {c }}$ [Figs. 2(e) and 2(f)], consistent with the characteristic of the SPE. Importantly, when $H$ is reversed, no $A$ change and clear $\phi$ reversal were observed, where the $\phi$ values are $\sim 180^{\circ}$ [see the thermal images at $H=-10 \mathrm{kOe}(\mathbf{H} \|-x$ direction $)$ in Figs. 2(c) and 2(d) and the $J_{\mathrm{c}}$ dependence of $A$ and $\phi$ in Figs. 2(e) and 2(f)]. The $H$ dependence of the $A$ and $\phi$ signals reflects the $M$ - $H$ curve of the $\left[\mathrm{Pt} / \mathrm{Fe}_{3} \mathrm{O}_{4}\right] \times 6$ sample [Figs. $2(\mathrm{~g})$ and $\left.2(\mathrm{~h})\right]$, indicating that the sign of the current-induced temperature modulation on the surface of the sample is reversed in response to the $\mathbf{M}$ reversal. This result confirms the spin origin of the observed temperature modulation. The signal is almost independent of the lock-in frequency $f$ (see Appendix A); this behavior is also one of the features of the SPE, which arises as a consequence of the temperature modulation confined near the interfaces, as demonstrated by the previous SPE measurements using Pt/YIG systems [31]. We also checked that the sign of the current-induced temperature modulation in the $\left[\mathrm{Pt} / \mathrm{Fe}_{3} \mathrm{O}_{4}\right] \times 6$ sample is the same as that of the SPE signals in the Pt/YIG systems [31]. Judging from the large difference in the electrical conductivity between $\mathrm{Pt}$ and $\mathrm{Fe}_{3} \mathrm{O}_{4}$ and the experimental results on the SSE in similar $\mathrm{Pt} / \mathrm{Fe}_{3} \mathrm{O}_{4}$ films [16,33], we can neglect the contribution coming from the anomalous Ettingshausen effect [43] in the $\mathrm{Fe}_{3} \mathrm{O}_{4}$ layers. Therefore, we conclude that the observed temperature modulation originates from the SPE in the $\left[\mathrm{Pt} / \mathrm{Fe}_{3} \mathrm{O}_{4}\right] \times 6$ sample.

Next, to investigate the $n$ dependence of the SPE signals, we performed the same experiments using the $\left[\mathrm{Pt} / \mathrm{Fe}_{3} \mathrm{O}_{4}\right] \times n$ samples with different $n$ values. We observed clear SPE signals in all the samples and found that the magnitude of the SPE signals $A$ is dramatically enhanced with increasing $n$ [compare the thermal images for $n=6$ and 1 shown in Figs. 3(b) and 3(c), respectively]. To quantitatively compare the SPE signals in the $\left[\mathrm{Pt} / \mathrm{Fe}_{3} \mathrm{O}_{4}\right] \times n$ samples, we plot $A$ at $H=+10 \mathrm{kOe}$ as a function of the charge current density, given by the charge current over the total cross-sectional area of the Pt layers, $j_{\mathrm{c}} \equiv J_{\mathrm{c}} /\left(w n t_{\mathrm{P}}\right)$ [Fig. 3(a)]. As shown in the inset in Fig. 3(a), the SPE magnitude per unit chargecurrent density $\Pi_{\mathrm{SPE}}(n) \equiv A / j_{\mathrm{c}}$ monotonically increases with increasing $n$, and the enhancement ratio between $n=12$ and $1, \Pi_{\mathrm{SPE}}(n=12) / \Pi_{\mathrm{SPE}}(n=1)$, reaches $\sim 40$. This SPE enhancement is almost consistent with the SSE enhancement in the $\left[\mathrm{Pt} / \mathrm{Fe}_{3} \mathrm{O}_{4}\right] \times n$ multilayer films (see [44]) but cannot be explained simply by regarding the multilayer films as plain stacks of independent $\mathrm{Pt} / \mathrm{Fe}_{3} \mathrm{O}_{4}$ bilayers, where the enhancement ratio is calculated to be $2 n-1$ (=23 for the $\left[\mathrm{Pt} / \mathrm{Fe}_{3} \mathrm{O}_{4}\right] \times 12$ sample; see Appendix B). Therefore, an unconventional mechanism is required to explain the significant enhancement of the SPE observed in the $\left[\mathrm{Pt} / \mathrm{Fe}_{3} \mathrm{O}_{4}\right] \times n$ multilayer films.

\section{B. Calculation of spin-current profile in multilayers}

We found that the observed enhancement of the SPE can be reproduced by introducing the magnon spin current in $\mathrm{F}$ and its connection with the electron spin current in $\mathrm{P}$ at the $\mathrm{P} / \mathrm{F}$ interfaces, as in the case of the SSE in multilayer films [33]. In the SPE, the electron spin current is driven by the charge current via the SHE in $\mathrm{P}$, and the magnitude of the temperature modulation is determined by the number of spins injected into $\mathrm{F}$ [28], which is the magnitude of the magnon spin current at the $\mathrm{P} / \mathrm{F}$ interfaces. In the plain $\mathrm{P} / \mathrm{F}$ bilayer system, the efficiency for generating the magnon spin current 
(a)
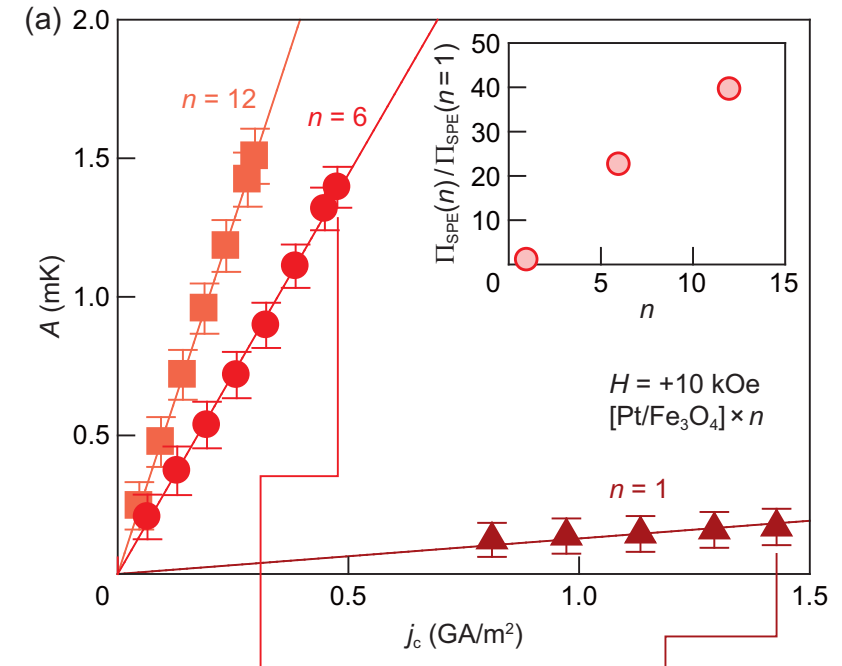

(b)
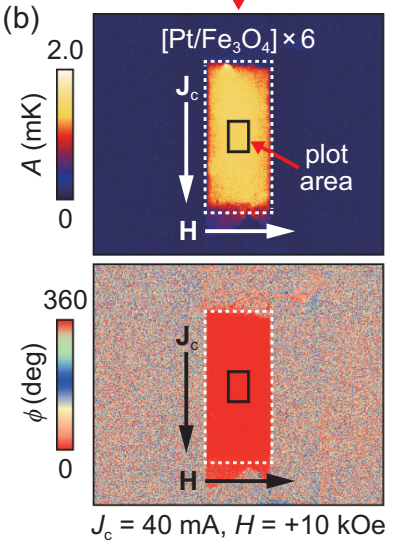
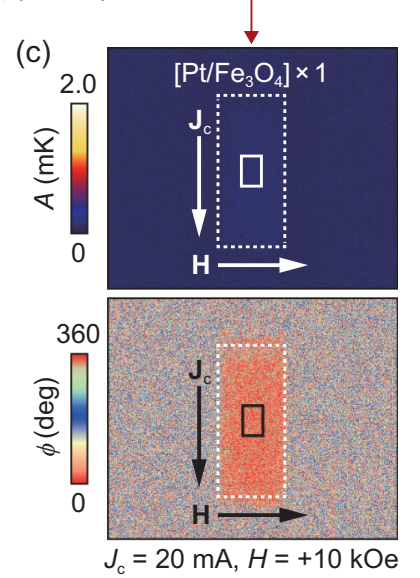

FIG. 3. (a) Charge-current density $j_{\mathrm{c}}$ dependence of $A$ for the $\left[\mathrm{Pt} / \mathrm{Fe}_{3} \mathrm{O}_{4}\right] \times n$ samples with $n=1,6$, and 12 at $H=+10 \mathrm{kOe}$, where $j_{\mathrm{c}} \equiv J_{\mathrm{c}} /\left(w n t_{\mathrm{P}}\right)$, with $w$ and $t_{\mathrm{P}}$ respectively being the width of the samples and the thickness of the Pt layer. The inset shows the $n$ dependence of $\Pi_{\mathrm{SPE}}(n) / \Pi_{\mathrm{SPE}}(n=1)$ for the $\left[\mathrm{Pt} / \mathrm{Fe}_{3} \mathrm{O}_{4}\right] \times n$ samples, where $\Pi_{\mathrm{SPE}}(n)$ is defined as $A / j_{\mathrm{c}}$ at $H=+10 \mathrm{kOe}$. (b) $A$ and $\phi$ images for the $\left[\mathrm{Pt} / \mathrm{Fe}_{3} \mathrm{O}_{4}\right] \times 6$ sample at $J_{\mathrm{c}}=40 \mathrm{~mA}$ $\left(j_{\mathrm{c}}=0.48 \mathrm{GA} / \mathrm{m}^{2}\right)$ and $H=+10 \mathrm{kOe}$. (c) $A$ and $\phi$ images for the $\left[\mathrm{Pt} / \mathrm{Fe}_{3} \mathrm{O}_{4}\right] \times 1$ sample at $J_{\mathrm{c}}=20 \mathrm{~mA}\left(j_{\mathrm{c}}=1.43 \mathrm{GA} / \mathrm{m}^{2}\right)$ and $H=+10 \mathrm{kOe}$.

by the SHE-driven electron spin current is limited because a back flow is induced to satisfy the boundary condition that the magnon spin current should vanish at the bottom surface [see the spin-current profile for $n=1$ in Fig. 4(a)]. In contrast, in the multilayer systems, such back flow is not induced but reused as the magnon spin current in the neighboring $\mathrm{F}$ layers owing to the connection between the magnon and electron spin currents, resulting in improved spin injection efficiency [see the spin-current profiles for $n \geqslant 2$ in Fig. 4(a)]. As the observable temperature modulation in the multilayer systems can be regarded as the integral of each layer's modulation, the increased efficiency at the inner interfaces results in enhanced temperature modulation in the multilayer systems.

To estimate the enhancement, we calculate the sum of the spin-current density at the interfaces, which is defined as $J_{\mathrm{s} \text {, sum }}^{\mathrm{int}}$, by computing the profiles of the magnon spin-current density $j_{\mathrm{m}}$ and the electron spin-current density $j_{\mathrm{s}}$ based on magnon

and electron-spin diffusion equations. We regard the system as one-dimensional along the thickness direction (the $z$ axis) and consider only spin currents polarizing in the field direction (the $x$ axis). The interface between the substrate and the bottom $\mathrm{F}$ layer is at $z=0$, and the surface of the top $\mathrm{P}$ layer is at $z=n\left(t_{\mathrm{F}}+t_{\mathrm{P}}\right) \equiv t_{\mathrm{ML}}$. Under the charge current along the $-y$ direction with the density $j_{\mathrm{c}}$ in $\mathrm{P}$, the spin-current density $j_{\mathrm{s}}$ is given by

$$
j_{\mathrm{s}}(z)=-\frac{\sigma_{\mathrm{s}}}{e} \frac{\partial}{\partial z} \mu_{\mathrm{s}}(z)+\theta_{\mathrm{SHE}} j_{\mathrm{c}}
$$

where $e, \sigma_{\mathrm{s}}, \mu_{\mathrm{s}}$, and $\theta_{\mathrm{SHE}}$ denote the elementary charge, spin conductivity, spin accumulation (in $\mathrm{eV}$ ), and spin Hall angle of $\mathrm{P}$, respectively [3]. When we assume that the magnon current is driven by magnon chemical potential [45], the magnon current density $j_{\mathrm{m}}$ is given by

$$
j_{\mathrm{m}}(z)=-\frac{\sigma_{\mathrm{m}}}{e} \frac{\partial}{\partial z} \mu_{\mathrm{m}}(z),
$$

where $\sigma_{\mathrm{m}}$ and $\mu_{\mathrm{m}}$ denote the magnon conductivity and magnon chemical potential (in $\mathrm{eV}$ ), respectively. We note that the derivation of the SPE enhancement discussed here does not necessarily require the magnon chemical potential as the driving force of $j_{\mathrm{m}}$; the magnon particle accumulation picture [27] also works. Here, we assume Eq. (2) as it is effective to describe the long-range transport of spins at room temperature and the transport coefficients are available [45]. The profile is obtained by solving the diffusion equation for magnons $(\alpha=\mathrm{m})$ and electron spins $(\alpha=\mathrm{s})$,

$$
\frac{\partial}{\partial \tilde{z}^{2}} \mu_{\alpha}(\tilde{z})=\frac{1}{\lambda_{\alpha}^{2}} \mu_{\alpha}(\tilde{z}),
$$

where $\lambda_{\alpha}$ denotes the decay length of $\mu_{\alpha}$ and $\tilde{z}$ represents a relative position in each layer $\left[0 \leqslant \tilde{z} \leqslant t_{\mathrm{F}}\left(t_{\mathrm{P}}\right)\right.$ for the $\mathrm{F}(\mathrm{P})$ layer] $[3,45]$. The boundary conditions at the top and bottom surfaces are $j_{\mathrm{s}}\left(t_{\mathrm{ML}}\right)=0$ and $j_{\mathrm{m}}(0)=0$. At the inner interfaces, $j_{\mathrm{m}}$ and $j_{\mathrm{s}}$ are continuous by assuming the reciprocal conversion between $j_{\mathrm{m}}$ and $j_{\mathrm{s}}[33,45]$, and the magnitude at the $\mathrm{P} / \mathrm{F}$ interfaces is given by

$$
j_{\alpha}=\frac{g_{\mathrm{s}}}{e}\left(\mu_{\mathrm{m}}-\mu_{\mathrm{s}}\right),
$$

with conversion efficiency $g_{\mathrm{s}}$. In our calculation, $\sigma_{\mathrm{s}}=1.3 \times$ $10^{6} \Omega^{-1} \mathrm{~m}^{-1}$ [33], $\sigma_{\mathrm{m}}=5 \times 10^{5} \Omega^{-1} \mathrm{~m}^{-1}[45], \lambda_{\mathrm{s}}=3 \mathrm{~nm}$ [39], $\lambda_{\mathrm{m}}=17 \mathrm{~nm}$ [20], and $g_{\mathrm{s}}=9.6 \times 10^{13} \Omega^{-1} \mathrm{~m}^{-2}$ [45] are used, where $\sigma_{\mathrm{m}}$ and $g_{\mathrm{s}}$ are assumed to be the same values for the Pt/YIG systems [45].

Figure 4(a) shows the $j_{\mathrm{m}}$ and $j_{\mathrm{s}}$ profiles along the thickness (z) direction for the $[\mathrm{P} / \mathrm{F}] \times n$ systems, with $n=1,2,6$, and 12. As expected, with increasing $n$, the number of interfaces with larger spin currents increases because of the series connection effect for the spin currents. This is the additional contribution to the SPE enhancement in the multilayers, which explains the deviation from the factor of $2 n-1$. Figure 4(b) shows the normalized $J_{\text {s, sum }}^{\text {int }}$ values as a function of $n$. The calculation shows that $J_{\mathrm{s}, \mathrm{sum}}^{\mathrm{int}}(n=12) / J_{\mathrm{s}, \mathrm{sum}}^{\mathrm{int}}(n=1)$ reaches $\sim 42$, which is consistent with the observed SPE enhancement [Fig. 4(b)] [note that the $n$ dependence of $J_{\text {s, sum }}^{\text {int }}$ can be compared with the experimental results in the inset in Fig. 3(a) because the observable temperature modulation 

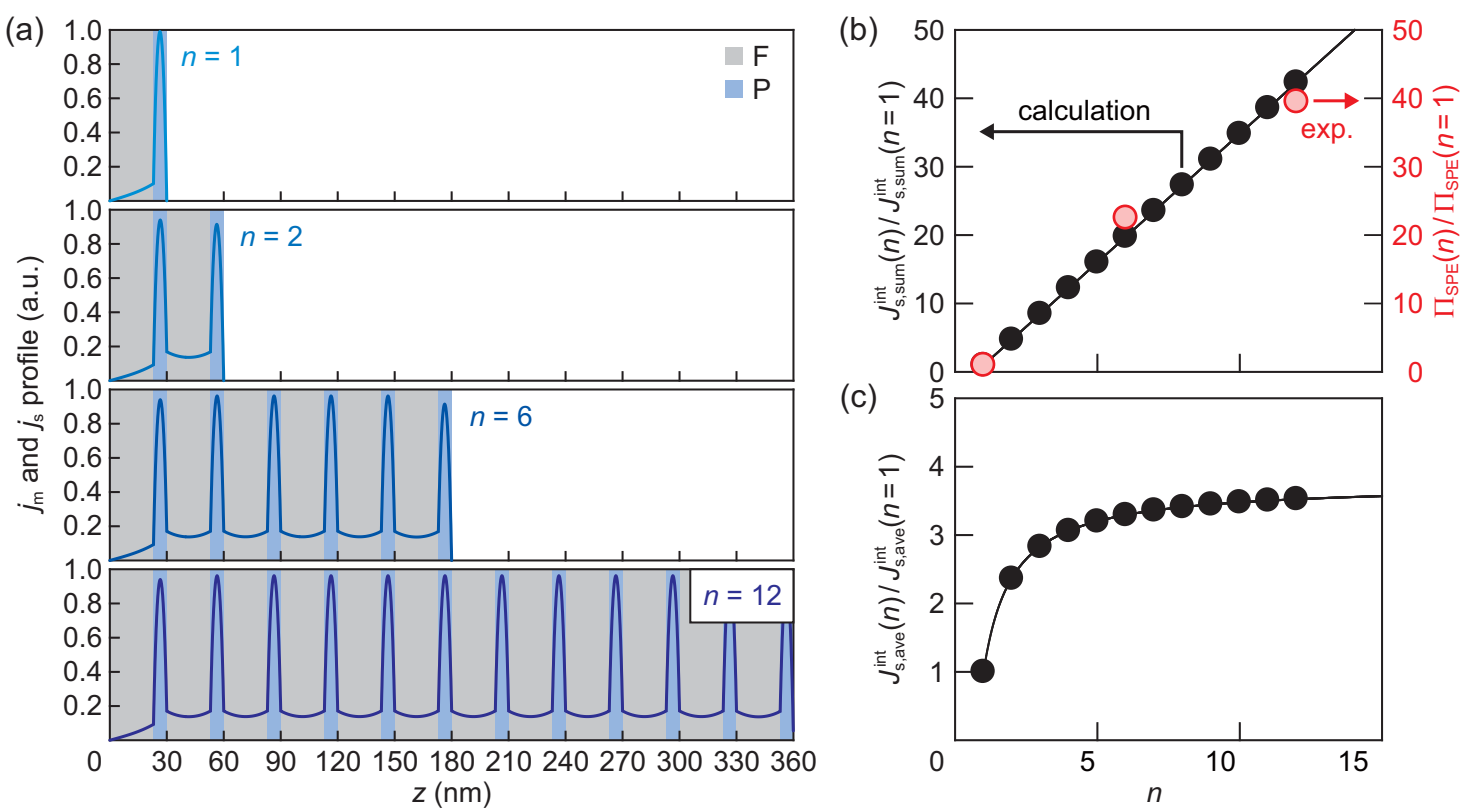

FIG. 4. (a) Calculated spin-current profile in the $[\mathrm{P} / \mathrm{F}] \times n$ multilayer films for various values of $n$. The profile consists of the $j_{\mathrm{m}}$ distribution in $\mathrm{F}$ and the $j_{\mathrm{s}}$ distribution in $\mathrm{P}$, where $j_{\mathrm{m}(\mathrm{s})}$ is the magnon (electron) spin-current density and $j_{\mathrm{m}}=j_{\mathrm{s}}$ at the P/F interfaces. (b) Calculated $n$ dependence of $J_{\mathrm{s}, \text { sum }}^{\mathrm{int}}(n) / J_{\mathrm{s} \text {, sum }}^{\mathrm{int}}(n=1)$, where $J_{\mathrm{s} \text {,sum }}^{\text {int }}$ is the sum of the spin-current density at the interfaces, and comparison with the observed $\Pi_{\mathrm{SPE}}(n) / \Pi_{\mathrm{SPE}}(n=1)$ values for the $\left[\mathrm{Pt} / \mathrm{Fe}_{3} \mathrm{O}_{4}\right] \times n$ systems. The experimental data are taken from the inset in Fig. 3(a). (c) Calculated $n$ dependence of $J_{\mathrm{s}, \text { ave }}^{\text {int }}(n) / J_{\mathrm{s}, \text { ave }}^{\text {int }}(n=1)$, where $J_{\mathrm{s}, \text { ave }}^{\text {int }}=J_{\mathrm{s}, \text { sum }}^{\text {int }} / n$.

in our experiments can be regarded as the integral of each layer's modulation]. In contrast, we also found that the spin-current density averaged by the number of $\mathrm{P} / \mathrm{F}$ bilayers is monotonically enhanced but gradually saturated with respect to $n$, as shown in Fig. 4(c), where $J_{\mathrm{s} \text {, ave }}^{\text {int }}\left(=J_{\mathrm{s}, \text { sum }}^{\text {int }} / n\right)$ normalized by the value at $n=1$ is plotted as a function of $n$ (see also [44]).

Finally, we mention the length scale of the SPE enhancement in the multilayer systems. In the above calculations, we assume the situation where $\lambda_{\mathrm{m}}<t_{\mathrm{F}}$ and $\lambda_{\mathrm{s}}<t_{\mathrm{P}}$ to evaluate the spin-current profiles, where the SPE enhancement is determined by the spin-current redistribution mainly affected by the adjacent layers. In contrast, with increasing $\lambda_{\mathrm{m}}$ and $\lambda_{\mathrm{s}}$ or decreasing $t_{\mathrm{F}}$ and $t_{\mathrm{P}}$, stronger SPE enhancement characterized by a new length scale can be expected, as discussed in the SSE study using multilayers [33]. Therefore, the determination of optimum thicknesses of each layer and the optimum $\mathrm{P} / \mathrm{F}$ material combination is important to realize efficient heatenergy control based on the SPE in multilayer systems.

\section{CONCLUSION}

In this study, we reported the observation of the spin Peltier effect (SPE) in alternately stacked $\mathrm{Pt} / \mathrm{Fe}_{3} \mathrm{O}_{4}$ multilayer films with various layer numbers. The temperature modulation on the surface of the multilayer films induced by the SPE was measured by means of the lock-in-thermography technique. The experimental results show that the SPE signals are significantly and monotonically enhanced with increasing the number of $\mathrm{Pt} / \mathrm{Fe}_{3} \mathrm{O}_{4}$ bilayers. The layer-number dependence of the SPE is similar to that of the spin Seebeck effect (SSE) in $\mathrm{Pt} / \mathrm{Fe}_{3} \mathrm{O}_{4}$ multilayer films, implying a reciprocal relation between the SPE and SSE. In fact, the SPE enhancement observed here can be explained by a mechanism similar to that proposed for explaining the enhancement of the SSE in multilayer systems; the essence of the SPE-enhancement mechanism is the redistribution of magnon and electron spin currents flowing normal to the multilayers due to their interconnection. Although the temperature modulation induced by the SPE is still very small, the experiments and calculations reported here provide guidelines for improving the thermospin conversion performance of the SPE and pave the way for realizing spin-current-driven thermal management technologies.

\section{ACKNOWLEDGMENTS}

This work was supported by PRESTO "Phase Interfaces for Highly Efficient Energy Utilization" (Grant No. JPMJPR12C1) and ERATO "Spin Quantum Rectification Project” (Grant No. JPMJER1402) from JST, Japan; Grantin-Aid for Scientific Research (A) (Grant No. JP15H02012) and Grant-in-Aid for Scientific Research on Innovative Area, "Nano Spin Conversion Science" (Grant No. JP26103005), from JSPS KAKENHI, Japan; the NEC Corporation; the Noguchi Institute; E-IMR, Tohoku University; H2020-MSCARISE-2016 SPICOLOST (Grant No. 734187); the Spanish Ministry of Economy and Competitiveness (Grant No. MAT2014-51982-C-R, including FEDER), Spain; and the Aragon regional government (E26), Spain. S.D. is supported by JSPS through a research fellowship for young scientists (Grant No. JP16J02422).

\section{APPENDIX A: LOCK-IN FREQUENCY DEPENDENCE OF THERMAL IMAGES}

LIT images include not only the magnitude and sign information of the temperature modulation but also the 
(a)

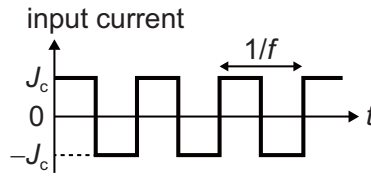

output thermal images

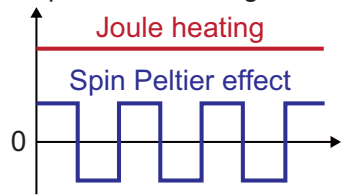

(c)
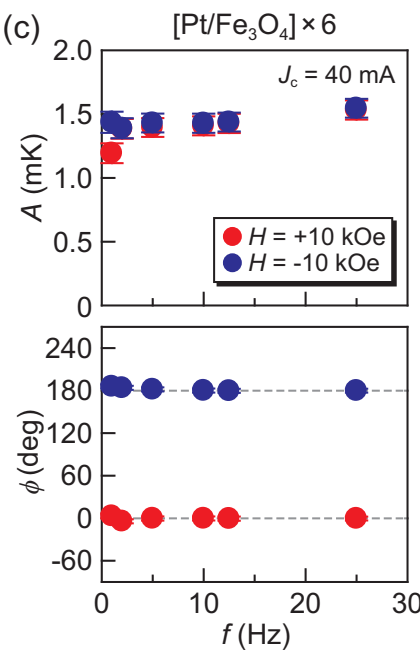

(b)

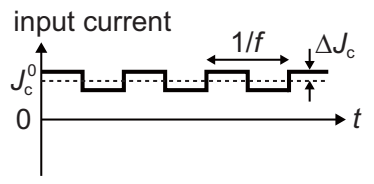

output thermal images

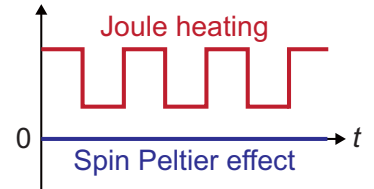

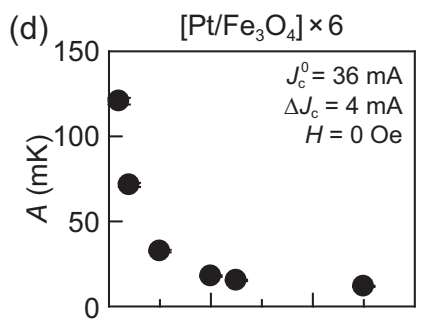

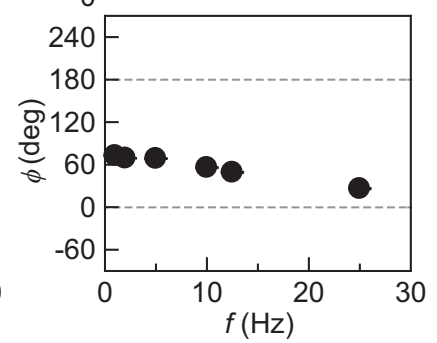

FIG. 5. (a) Condition for measuring the SPE by means of LIT. (b) Condition for measuring Joule heating by means of LIT. In the Jouleheating measurements, a dc offset of $J_{\mathrm{c}}^{0}$ and an ac charge current with rectangular wave modulation with the amplitude $\Delta J_{\mathrm{c}}$ and frequency $f$ are applied to the sample. In this condition, although both the SPE and Joule-heating signals appear in the first harmonic response of the thermal images, the observed LIT images are governed by the Joule-heating-induced temperature modulation because it is much greater than the SPE signal. (c) $f$ dependence of $A$ and $\phi$ for the $\left[\mathrm{Pt} / \mathrm{Fe}_{3} \mathrm{O}_{4}\right] \times 6$ sample in the SPE condition at $J_{\mathrm{c}}=40 \mathrm{~mA}$ and $H=$ +10 or $-10 \mathrm{kOe}$. (d) $f$ dependence of $A$ and $\phi$ for the $\left[\mathrm{Pt} / \mathrm{Fe}_{3} \mathrm{O}_{4}\right] \times 6$ sample in the Joule-heating condition at $J_{\mathrm{c}}^{0}=36 \mathrm{~mA}, \Delta J_{\mathrm{c}}=4 \mathrm{~mA}$, and $H=0$ Oe.

temporal information, i.e., the time delay due to thermal diffusion [41]. Therefore, by measuring the $f$ dependence of LIT images, one can obtain the time scale for reaching steady states, where the LIT images at lower $f$ values are closer to temperature distribution at steady-state conditions. Previous experiments using Pt/YIG systems show that the SPE signals are independent of $f$, at least, in the range of $1 \leqslant f \leqslant 25 \mathrm{~Hz}$ [31,32], meaning that the temperature modulation induced by the SPE immediately reaches the steady state. This is because the SPE-induced temperature modulation is confined only in the vicinity of the $\mathrm{P} / \mathrm{F}$ interfaces, which is quite different from thermal diffusion expected from conventional heat sources, such as Joule heating [31,32].

To demonstrate this unique feature of the SPE in multilayer films, we measured the $f$ dependence of the $A$ and $\phi$ images by using the $\left[\mathrm{Pt} / \mathrm{Fe}_{3} \mathrm{O}_{4}\right] \times 6$ sample. In Fig. 5(c), we show the $A$ and $\phi$ values on the sample surface at $J_{\mathrm{c}}=40 \mathrm{~mA}$ as a function of $f$, measured when $H=+10$ or $-10 \mathrm{kOe}$. We confirmed that the magnitude of $A$ is nearly constant at all $f$ values, which is in sharp contrast to the $f$ dependence of Joule heating in the same sample [Fig. 5(d)], where the Joule-heating signal monotonically increases with decreasing $f$ due to thermal diffusion [note that the Joule heating can be measured by applying a dc offset to the rectangular ac current, although it is eliminated in the SPE measurements because of the zero offset, as depicted in Figs. 5(a) and 5(b)]. We observed similar behavior also in the $\left[\mathrm{Pt} / \mathrm{Fe}_{3} \mathrm{O}_{4}\right] \times 1$ and $\left[\mathrm{Pt} / \mathrm{Fe}_{3} \mathrm{O}_{4}\right] \times 12$ samples. The weak $f$ dependence of the temperature modulation supports our interpretation that the observed signals in Figs. 2 and 3 are attributed to the SPE.

\section{APPENDIX B: CALCULATIONS FOR A PLAIN STACK OF INDEPENDENT P/F BILAYERS}

Here, we show that the observed enhancement of the SPE in the $\left[\mathrm{Pt} / \mathrm{Fe}_{3} \mathrm{O}_{4}\right] \times n$ multilayer films cannot be explained simply by stacking independent $\mathrm{Pt} / \mathrm{Fe}_{3} \mathrm{O}_{4}$ bilayers by means of numerical calculations based on a two-dimensional finiteelement method. By solving a standard heat diffusion equation using the COMSOL MULTIPHYSICS software, we calculated the steady-state cross-sectional temperature distribution in the $z-x$ plane in the model system consisting of a $\left[\mathrm{Pt} / \mathrm{Fe}_{3} \mathrm{O}_{4}\right] \times n$ multilayer film formed on a $\mathrm{MgO}$ substrate with different $n$ values. The dimensions of the model systems are set to be the same as those of the samples used for the experiments; the thicknesses of $\mathrm{Pt}, \mathrm{Fe}_{3} \mathrm{O}_{4}$, and $\mathrm{MgO}$ are $7 \mathrm{~nm}, 23 \mathrm{~nm}$, and 0.5 $\mathrm{mm}$, respectively, and the width of all the layers is $2 \mathrm{~mm}$. As demonstrated in Ref. [31], the temperature distribution induced by the SPE can be reproduced by assuming the presence of a dipolar heat source, a symmetric pair of positive and negative heat-source components, near the $\mathrm{P} / \mathrm{F}$ interface. In the present model systems, we put a dipolar heat source on each $\mathrm{Pt} / \mathrm{Fe}_{3} \mathrm{O}_{4}$ interface, where one of the heat-source components is placed on $\mathrm{Pt}$ and the other is on $\mathrm{Fe}_{3} \mathrm{O}_{4}$ [Fig. 6(a)]. The polarity of the dipolar heat source on each interface is determined according to the direction of the spin currents, where the sign of the heat-source component on the top Pt layer is set to be positive. We assume that the thickness of the heat-source components is half of the thickness of each layer: $3.5 \mathrm{~nm}(11.5$ $\mathrm{nm})$ for the component on the $\mathrm{Pt}\left(\mathrm{Fe}_{3} \mathrm{O}_{4}\right)$ layers. The width of the dipolar heat sources is the same as that of the Pt and $\mathrm{Fe}_{3} \mathrm{O}_{4}$ layers $(2 \mathrm{~mm})$. This model represents the plain stack of the independent $\mathrm{Pt} / \mathrm{Fe}_{3} \mathrm{O}_{4}$ bilayers. As a boundary condition, the temperature of the bottom of the $\mathrm{MgO}$ substrate is fixed at $300 \mathrm{~K}$, and the top and side surfaces of the systems are connected to air. The thermal conductivities of $\mathrm{Pt}, \mathrm{Fe}_{3} \mathrm{O}_{4}$, and $\mathrm{MgO}$ are assumed to be the values shown in Refs. $[18,46]$. The interfacial thermal conductance at the $\mathrm{Pt} / \mathrm{Fe}_{3} \mathrm{O}_{4}$ and $\mathrm{Fe}_{3} \mathrm{O}_{4} / \mathrm{MgO}$ interfaces (the heat-transfer coefficient from the system to air) is set to be $1.0 \times 10^{8} \mathrm{~W} / \mathrm{m}^{2} \mathrm{~K}\left(10 \mathrm{~W} / \mathrm{m}^{2} \mathrm{~K}\right)$. In this condition, we calculated the temperature difference $\Delta T$ distribution for various values of $n$, where $\Delta T$ is defined as the difference from $300 \mathrm{~K}$.

In Fig. 6(b), we show the calculated $\Delta T$ distribution in the $z-x$ plane and the $\Delta T$ profile along the $z$ direction for the $\left[\mathrm{Pt} / \mathrm{Fe}_{3} \mathrm{O}_{4}\right] \times 6$ multilayer film, where $x=0$ is at the 
(a)

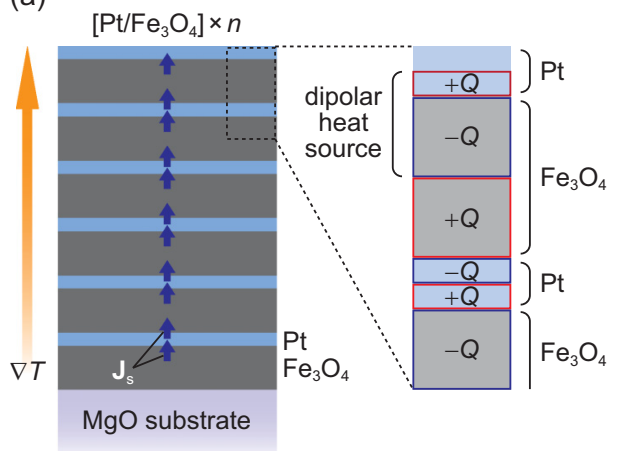

(b)

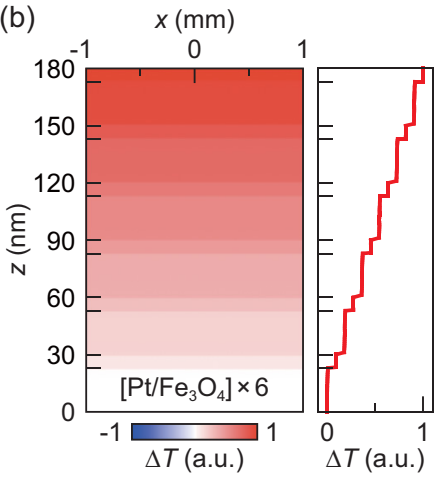

(c)

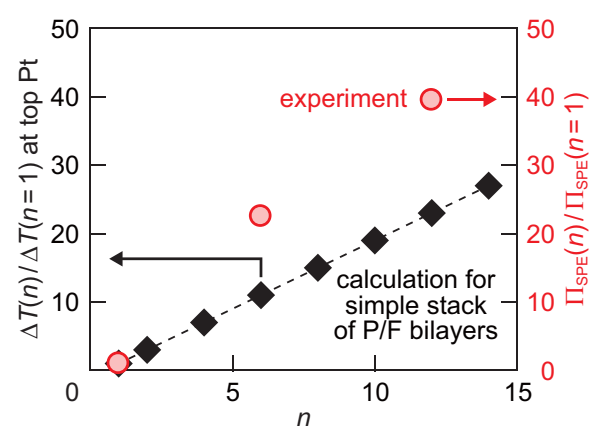

FIG. 6. (a) A schematic illustration of the model system used for calculating the temperature distribution in a plain stack of independent $\mathrm{P} / \mathrm{F}$ bilayers. The model system consists of a $\left[\mathrm{Pt} / \mathrm{Fe}_{3} \mathrm{O}_{4}\right] \times n$ multilayer film formed on a $\mathrm{MgO}$ substrate. A dipolar heat source comprising a symmetric pair of a positive component $+Q$ and a negative component $-Q$ is placed on each $\mathrm{Pt} / \mathrm{Fe}_{3} \mathrm{O}_{4}$ interface. The polarity of the dipolar heat sources is determined according to the direction of the spin currents. (b) Calculated temperature difference $\Delta T$ distribution in the $z-x$ plane and $\Delta T$ profile along the $z$ direction for the $\left[\mathrm{Pt} / \mathrm{Fe}_{3} \mathrm{O}_{4}\right] \times 6$ model system shown in (a). $\Delta T$ is the temperature difference from $300 \mathrm{~K}$. (c) Comparison of the $n$ dependence between the calculated $\Delta T(n) / \Delta T(n=1)$ values at the top Pt layer and the observed $\Pi_{\mathrm{SPE}}(n) / \Pi_{\mathrm{SPE}}(n=1)$ values for the $\left[\mathrm{Pt} / \mathrm{Fe}_{3} \mathrm{O}_{4}\right] \times n$ systems. The experimental data are taken from the inset in Fig. 3(a).

center of the sample and $z=0$ is at the bottom $\mathrm{Fe}_{3} \mathrm{O}_{4} / \mathrm{MgO}$ interface. The $\Delta T$ values increase along the $z$ direction with stacking the $\mathrm{Pt} / \mathrm{Fe}_{3} \mathrm{O}_{4}$ bilayers. The discontinuous jump at each $\mathrm{Pt} / \mathrm{Fe}_{3} \mathrm{O}_{4}$ interface appears as a result of the interfacial thermal resistance. We obtained similar $\Delta T$ distributions for all the $\left[\mathrm{Pt} / \mathrm{Fe}_{3} \mathrm{O}_{4}\right] \times n$ model systems.

Figure 6(c) shows the $\Delta T$ values, normalized by the value at $n=1$, at the top Pt layer as a function of $n$ for the $\left[\mathrm{Pt} / \mathrm{Fe}_{3} \mathrm{O}_{4}\right] \times n$ systems. We found that the enhancement ratio of $\Delta T$ in these systems is calculated to be $2 n-1$; $\Delta T$ is simply proportional to the $\mathrm{Pt} / \mathrm{Fe}_{3} \mathrm{O}_{4}$-interface number. This enhancement ratio is much smaller than the observed SPE enhancement as compared in Fig. 6(c), suggesting the importance of the interlayer interaction. In Sec. III B, we discussed the interlayer interaction in terms of the spin-current redistribution.
[1] S. A. Wolf, D. D. Awschalom, R. A. Buhrman, J. M. Daughton, S. von Molnar, M. L. Roukes, A. Y. Chtchelkanova, and D. M. Treger, Spintronics: A spin-based electronics vision for the future, Science 294, 1488 (2001).

[2] I. Žutić, J. Fabian, and S. Das Sarma, Spintronics: Fundamentals and applications, Rev. Mod. Phys. 76, 323 (2004).

[3] Spin Current, edited by S. Maekawa, E. Saitoh, S. O. Valenzuela, and T. Kimura (Oxford University Press, Oxford, 2012).

[4] S. Maekawa, H. Adachi, K. Uchida, J. Ieda, and E. Saitoh, Spin current: Experimental and theoretical aspects, J. Phys. Soc. Jpn. 82, 102002 (2013).

[5] C. Wood, Materials for thermoelectric energy conversion, Rep. Prog. Phys. 51, 459 (1988).

[6] S. B. Riffat and X. Ma, Thermoelectrics: A review of present and potential applications, Appl. Therm. Eng. 23, 913 (2003).

[7] X. Zhang and L.-D. Zhao, Thermoelectric materials: Energy conversion between heat and electricity, J. Materiomics 1, 92 (2015).

[8] K. Uchida, S. Takahashi, K. Harii, J. Ieda, W. Koshibae, K. Ando, S. Maekawa, and E. Saitoh, Observation of the spin Seebeck effect, Nature (London) 455, 778 (2008).

[9] K. Uchida, J. Xiao, H. Adachi, J. Ohe, S. Takahashi, J. Ieda, T. Ota, Y. Kajiwara, H. Umezawa, H. Kawai, G. E. W. Bauer, S. Maekawa, and E. Saitoh, Spin Seebeck insulator, Nat. Mater. 9, 894 (2010)

[10] C. M. Jaworski, J. Yang, S. Mack, D. D. Awschalom, J. P. Heremans, and R. C. Myers, Observation of the spin-Seebeck effect in a ferromagnetic semiconductor, Nat. Mater. 9, 898 (2010).

[11] K. Uchida, H. Adachi, T. Ota, H. Nakayama, S. Maekawa, and E. Saitoh, Observation of longitudinal spin-Seebeck effect in magnetic insulators, Appl. Phys. Lett. 97, 172505 (2010).

[12] M. Weiler, M. Althammer, F. D. Czeschka, H. Huebl, M. S. Wagner, M. Opel, I.-M. Imort, G. Reiss, A. Thomas, R. Gross, and S. T. B. Goennenwein, Local Charge and Spin Currents in Magnetothermal Landscapes, Phys. Rev. Lett. 108, 106602 (2012).

[13] A. Kirihara, K. Uchida, Y. Kajiwara, M. Ishida, Y. Nakamura, T. Manako, E. Saitoh, and S. Yorozu, Spin-current-driven thermoelectric coating, Nat. Mater. 11, 686 (2012).

[14] D. Qu, S. Y. Huang, J. Hu, R. Wu, and C. L. Chien, Intrinsic Spin Seebeck Effect in Au/YIG, Phys. Rev. Lett. 110, 067206 (2013).

[15] T. Kikkawa, K. Uchida, Y. Shiomi, Z. Qiu, D. Hou, D. Tian, H Nakayama, X.-F. Jin, and E. Saitoh, Longitudinal Spin Seebeck Effect Free from the Proximity Nernst Effect, Phys. Rev. Lett. 110, 067207 (2013).

[16] R. Ramos, T. Kikkawa, K. Uchida, H. Adachi, I. Lucas, M. H. Aguirre, P. Algarabel, L. Morellón, S. Maekawa, E. Saitoh, and M. R. Ibarra, Observation of the spin Seebeck effect in epitaxial $\mathrm{Fe}_{3} \mathrm{O}_{4}$ thin films, Appl. Phys. Lett. 102, 072413 (2013).

[17] S. H. Wang, L. K. Zou, J. W. Cai, B. G. Shen, and J. R. Sun, Transverse thermoelectric effects in platinum strips on permalloy films, Phys. Rev. B 88, 214304 (2013). 
[18] K. Uchida, M. Ishida, T. Kikkawa, A. Kirihara, T. Murakami, and E. Saitoh, Longitudinal spin Seebeck effect: From fundamentals to applications, J. Phys. Condens. Matter 26, 343202 (2014).

[19] T. Kikkawa, K. Uchida, S. Daimon, Z. Qiu, Y. Shiomi, and E. Saitoh, Critical suppression of spin Seebeck effect by magnetic fields, Phys. Rev. B 92, 064413 (2015).

[20] A. Anadón, R. Ramos, I. Lucas, P. A. Algarabel, L. Morellón, M. R. Ibarra, and M. H. Aguirre, Characteristic length scale of the magnon accumulation in $\mathrm{Fe}_{3} \mathrm{O}_{4} / \mathrm{Pt}$ bilayer structures by incoherent thermal excitation, Appl. Phys. Lett. 109, 012404 (2016).

[21] K. Uchida, H. Adachi, T. Kikkawa, A. Kirihara, M. Ishida, S. Yorozu, S. Maekawa, and E. Saitoh, Thermoelectric generation based on spin Seebeck effects, Proc. IEEE 104, 1946 (2016); Erratum: Proc. IEEE 104, 1499 (2016).

[22] J. Xiao, G. E. W. Bauer, K. Uchida, E. Saitoh, and S. Maekawa, Theory of magnon-driven spin Seebeck effect, Phys. Rev. B 81, 214418 (2010).

[23] H. Adachi, J.-i. Ohe, S. Takahashi, and S. Maekawa, Linearresponse theory of spin Seebeck effect in ferromagnetic insulators, Phys. Rev. B 83, 094410 (2011).

[24] S. S.-L. Zhang and S. Zhang, Spin convertance at magnetic interfaces, Phys. Rev. B 86, 214424 (2012).

[25] H. Adachi, K. Uchida, E. Saitoh, and S. Maekawa, Theory of the spin Seebeck effect, Rep. Prog. Phys. 76, 036501 (2013).

[26] S. Hoffman, K. Sato, and Y. Tserkovnyak, Landau-Lifshitz theory of the longitudinal spin Seebeck effect, Phys. Rev. B 88, 064408 (2013).

[27] S. M. Rezende, R. L. Rodríguez-Suárez, R. O. Cunha, A. R. Rodrigues, F. L. A. Machado, G. A. Fonseca Guerra, J. C. Lopez Ortiz, and A. Azevedo, Magnon spin-current theory for the longitudinal spin-Seebeck effect, Phys. Rev. B 89, 014416 (2014).

[28] J. Flipse, F. K. Dejene, D. Wagenaar, G. E. W. Bauer, J. Ben Youssef, and B. J. van Wees, Observation of the Spin Peltier Effect for Magnetic Insulators, Phys. Rev. Lett. 113, 027601 (2014).

[29] V. Basso, E. Ferraro, A. Magni, A. Sola, M. Kuepferling, and M. Pasquale, Nonequilibrium thermodynamics of the spin Seebeck and spin Peltier effects, Phys. Rev. B 93, 184421 (2016).

[30] V. Basso, E. Ferraro, and M. Piazzi, Thermodynamic transport theory of spin waves in ferromagnetic insulators, Phys. Rev. B 94, 144422 (2016).

[31] S. Daimon, R. Iguchi, T. Hioki, E. Saitoh, and K. Uchida, Thermal imaging of spin Peltier effect, Nat. Commun. 7, 13754 (2016).

[32] S. Daimon, K. Uchida, R. Iguchi, T. Hioki, and E. Saitoh, Thermographic measurements of the spin Peltier effect in metal/yttrium-iron-garnet junction systems, arXiv:1705.02094.

[33] R. Ramos, T. Kikkawa, M. H. Aguirre, I. Lucas, A. Anadón, T. Oyake, K. Uchida, H. Adachi, J. Shiomi, P. A. Algarabel,
L. Morellón, S. Maekawa, E. Saitoh, and M. R. Ibarra, Unconventional scaling and significant enhancement of the spin Seebeck effect in multilayers, Phys. Rev. B 92, 220407(R) (2015).

[34] R. Ramos, A. Anadón, I. Lucas, K. Uchida, P. A. Algarabel, L. Morellón, M. H. Aguirre, E. Saitoh, and M. R. Ibarra, Thermoelectric performance of spin Seebeck effect in $\mathrm{Fe}_{3} \mathrm{O}_{4} / \mathrm{Pt}$ based thin film heterostructures, APL Mater. 4, 104802 (2016).

[35] R. Ramos, T. Kikkawa, A. Anadón, I. Lucas, K. Uchida, P. A. Algarabel, L. Morellón, M. H. Aguirre, E. Saitoh, and M. R. Ibarra, Temperature dependence of the spin Seebeck effect in $\left[\mathrm{Fe}_{3} \mathrm{O}_{4} / \mathrm{Pt}\right]_{n}$ multilayers, AIP Adv. 7, 055915 (2017).

[36] S. O. Valenzuela and M. Tinkham, Direct electronic measurement of the spin Hall effect, Nature (London) 442, 176 (2006).

[37] T. Kimura, Y. Otani, T. Sato, S. Takahashi, and S. Maekawa, Room-Temperature Reversible Spin Hall Effect, Phys. Rev. Lett. 98, 156601 (2007).

[38] A. Hoffmann, Spin Hall effects in metals, IEEE Trans. Magn. 49, 5172 (2013).

[39] J. Sinova, S. O. Valenzuela, J. Wunderlich, C. H. Back, and T. Jungwirth, Spin Hall effects, Rev. Mod. Phys. 87, 1213 (2015).

[40] Y. Tserkovnyak, A. Brataas, G. E. W. Bauer, and B. I. Halperin, Nonlocal magnetization dynamics in ferromagnetic heterostructures, Rev. Mod. Phys. 77, 1375 (2005).

[41] O. Breitenstein, W. Warta, and M. Langenkamp, Lock-in Thermography: Basics and Use for Evaluating Electronic Devices and Materials (Springer, Berlin, 2010).

[42] O. Wid, J. Bauer, A. Muller, O. Breitenstein, S. S. P. Parkin, and G. Schmid, Investigation of the unidirectional spin heat conveyer effect in a $200 \mathrm{~nm}$ thin yttrium iron garnet film, Sci. Rep. 6, 28233 (2016).

[43] S. R. Boona, R. C. Myers, and J. P. Heremans, Spin caloritronics, Energy Environ. Sci. 7, 885 (2014).

[44] In Fig. 3(a), the SPE is compared in terms of the magnitude of the output temperature difference per unit charge-current density. In contrast, in Ref. [33], the SSE is compared in terms of the magnitude of the output voltage per unit temperature gradient, where the SSE enhancement is saturated with increasing the number of layers. If we plot the temperature gradient per unit applied voltage, the output of the SPE is also saturated, and the enhancement factor is comparable to or slightly smaller than that of the SSE [see also Figs. 4(b) and 4(c)].

[45] L. J. Cornelissen, K. J. H. Peters, G. E. W. Bauer, R. A. Duine, and B. J. van Wees, Magnon spin transport driven by the magnon chemical potential in a magnetic insulator, Phys. Rev. B 94, 014412 (2016).

[46] G. A. Slack, Thermal conductivity of $\mathrm{MgO}, \mathrm{Al}_{2} \mathrm{O}_{3}, \mathrm{MgAl}_{2} \mathrm{O}_{4}$, and $\mathrm{Fe}_{3} \mathrm{O}_{4}$ crystals from $3^{\circ}$ to $300^{\circ} \mathrm{K}$, Phys. Rev. 126, 427 (1962). 